\title{
Polipropilen Blendlerinin Non-İzotermal Bozunma Kinetiği
}

\author{
Fatih Doğan \\ Çanakkale Onsekiz Mart Üniversitesi, Eğitim Fakültesi, Matematik ve Fen Bilimleri Eğitimi Bölümü, Kimya ABD, \\ 17100-Çanakkale, +90 2862180018 , \\ fatihdogan@comu.edu.tr
}

Geliş/Recieved: 2 Mart (March) 2017

Kabul/Accepted: 18 Nisan (April) 2017

DOI: $10.18466 /$ cbayarfbe.308937

\begin{abstract}
Özet
Bu çalışmada oksidatif polimerizasyon yöntemi ile sentezlenmiş olan sulfoaminonaftol polimerinin (6amino-4-hidroksi-2-naftalensülfonik asit, PSAN) polipropilen ile twin-screw microextruder'de çeşitli blendleri hazırlandı. Hazırlanan blendlerin 1sısal kararlılıkları ve katı hal bozunma kinetikleri incelendi. Tüm blendlerin termogramları tek basamaklı bir bozunma reaksiyonu sergiledi. Bununla birlikte ilave PSAN katkı polimerinin miktarı artıkça PSAN/polipropilen blendlerinin bozunmaya başlama sıcaklık değerlerinin yükselerek termal karalılığının artığı görüldü. Aynı zamanda PSAN/polipropilen blendlerinin farklı ısıtma hızlarında elde edilen DTG termogramları, maksimum reaksiyon hızına karşılık gelen sıcaklık değerlerinin ısıtma hızıyla doğru orantılı olduğunu gösterdi. PSAN/polipropilen blendlerinin isısal katı hal bozunma kinetiklerinin hesaplanmasında integral yöntemlerden Wanjun-Yuwen-Hen-Cunxin (WHYC), Madhusudanan-Krishnan-Ninan (MKN) ve Coats-Redfern (CR), diferansiyel yöntemlerden Horowitz-Metzger (HM) ve van Krevelen (vK) ve fark diferansiyel yöntemlerden MacCallum-Tanner (MT) yöntemi kullanıldı. Kinetik hesaplamalar PSAN/polipropilen blendlerinin ssısal olarak bozunmaya başlaması için gerekli olan aktivasyon enerjisinin, blendlerdeki PSAN polimerinin \% miktarına bağlı olduğunu gösterdi. Termodinamik parametreler ise bozunma reaksiyonunun normalden daha hızlı gerçekleştiğini açığa çıkardı.
\end{abstract}

Anahtar Kelimeler- Polipropilen, katı hal bozunma kinetiği, aktivasyon enerjisi

\section{Non-isothermal Decomposition Kinetic of Polypropylene Blends}

\begin{abstract}
A series of polypropylene (PP) blends was prepared by mixing (in twin-screw microextruder) the appropriate amount of sulphoamino naphtol polymer (PSAN) with commercially avaible PP. For this purpose, PSAN was synthesized by oxidative polymerization process in alkaline medium, in single step. The thermal stabilities and kinetics of solid state of the each blends were studied. TG analysis assigned the one-stage decomposition for each blends. DTG thermograms of blends showed that the maximumrates of decomposition increased by an increasing heating rate. The decomposition kinetics of blends were also studied by using integral methods: Wanjun-Yuwen-Hen-Cunxin (WHYC), Madhusudanan-Krishnan-Ninan (MKN), Coats and Redfern (CR), differential methods :Horowitz-Metzger (HM) and van Krevelen (vK) and difference-differential methods: MacCallumTanner (MT). Kinetic calculations showed values of activation energy required for thermal decomposition kinetics of blends was quite high and also increased with an amount of additive PSAN. Finally the thermodynamic parameters exhibited that the decomposition reaction occurred very faster than normal.
\end{abstract}

Keywords- Polypropylene, solid state decomposition kinetic, activation energy

\section{Giriş}

Poliolefin türevi malzemeler üretimleri, işlenmeleri veya kullanımları sırasında yüksek 1sı, nem, toz ve çeşitli kimyasallar gibi etkenlerle çok sık etkileşim halindedirler. Söz konusu etken parametreler poliolefin türevi malzemelerin fiziksel özelliklerini veya kimyasal yapılarını belirli düzeyde veya tamamen değiştirebilmektedirler. Bu nedenle son yıllarda teknoloji ve sanayinin hızla gelişmesi 
fiziksel, kimyasal ve mekaniksel olarak dayanıklı istenilen özellikli poliolefin temelli materyal ve kompozitlere olan ihtiyacı önemli ölçüde arttırmıştır. Günümüz teknolojisinde istenilen özellikli materyaller, poliolefinlerin içerisine çeşitli dolgu maddelerinin, plastikleştiricilerin, antioksidanların, daha yüksek sıcaklıklara kadar kullanımını sağlamak için 1sı stabilizatörlerinin, alevlenmeyi geciktiricilerin, renklendiricilerin, UVstabilizatörlerinin ve antistatik maddelerin belirli oranlarda katılması ile sağlanmaktadır. Bununla birlikte istenilen özellikli materyal eldesi ile birlikte işleme sırasındaki ısısal stabilite ve fizikomekaniksel özellikler gibi parametrelerde malzemenin endüstride kullanımında dikkate alınmaktadır. Bu kapsamda son zamanlarda poliolefinler üzerinde istenilen özelliklerin elde edilmesi amacıyla pek çok çalışma yapılmıştır. Şirin ve ark çeşitli çalışmalarında eriyik halde farklı tip poliolefin malzemeler (HYPE-PP) içerisine $\mathrm{CaCO}_{3}$ dolgu maddesini ilave ederek kullanılan poliolefinlerin fiziko-mekanik özelliklerini iyileştirmeyi ve termal stabilitelerini artırmayı amaçlamışlardır[1-4]. Bir başka çalışmada Zsoldos ve Kollárpolyolefin/polimetilmetakrilat blendlerinin yapısal özelliklerini ve ısısal stabilitelerini araştırmışlardır [5]. Buna göre poliolefinlerin polimetilmetakrilatlarda karışabilirliklerinin belirli oranlarda değiştiğini göstermişler ve kullanılan poliolefinlerin bazı fiziko-mekanik özelliklerinde önemli iyileştirmeler sağlamışlardır. Rogriga ve ark yüksek yoğunluklu (HYPE) ve düşük yoğunluklu (LYPE) polietilen malzemelerin içerisine birbirinden farklı biodegrade olabilen katkılar ilave ederek blendlerin 1sısal kararlılıklarını incelemişlerdir. Sonuçta biodegrade olabilen katkı miktarlarıyla poliolefinlerin ısısal stabilitelerinde önemli bir artış sağlamışlardır [6].Schartel ve ark, polipropilen (PP) içerisine flax ilavesinin PP'nin alev dayanıklılığını artığını rapor etmişlerdir [7]. Cai ve ark HYPE/parafin kompozitlerinin termal enerji depolama malzemeleri olabileceğini ileri sürerek termal stabilitenin enerji depolamada önemli bir parametre olduğunu ifade etmişlerdir [8]. Yapılan bu çalışmada ise oksidatif polimerizasyon yöntemi vasitasiyla daha önce sentezlenmiş sulfoaminonaftol polimeri ve polipropilenin üç farklı tip blendi eriyik halde twin-screw microextruder'de hazırlanmıştır. Daha sonra hazırlanan blendlerin ısısal kararlılıkları ve katı hal bozunma kinetiklerinin incelenmesi amaçlanmıştır.

\section{Materyal ve Metot}

\subsection{Materyal}

Oksidatif polimerizasyonda kullanılan sulfoaminonaftol monomeri, 6-amino-4-hidroksi-2naftalensülfonik asit [SAN] ( $\gamma$-gamma asit,\%98, ACS reagent) Sigma-Aldrich firmasindan alındı. Sodyumhipoklorit (\%30 luk sulu çözeltisi), hidrojen peroksit (\%30 luk sulu çözeltisi) ve amonyumperoksidisülfat ( $\geq \% 98$, ACS reagent) sirasiyla Paksoy Kimya, Merck ve Sigma-Aldrich firmalarından alınarak polimerizasyonlarda oksidant olarak kullanıldı. Hidroklorik asit (\%37, AR grade) Sigma-Aldrich firmasindan temin edilerek polimerlerin nötralleştirilmesinde kullanıldı. Potasyum hidroksit $(\geq \% 85$, ACS reagent, pellets) Sigma-Aldrich firmasından temin edilip polimerizasyon işlemlerinde sulu çözelti halinde kullanıldı. Asetonitril, metanol, etanol, kloroform, aseton, tetrahidrofuran, diklormetan ve diğer çözcüler Merck firmasından alınarak çözünürlük ve ortamda kalan monomerlerin uzaklaştılması işlemlerimlerinde kullanıldı. Filmlik polipropilen, FH-250 PETKİM A.Ş den temin edilerek blendlerin hazırlanmasında kullanıldı

\subsubsection{Sulfoaminonaftol monomerinin polimerizasyonu}

[SAN]

SAN monomeri $\mathrm{NaOCl}$ ile sulu ortamda oksidatif polikondenzasyon yöntemiyle sentezlendi. Üç farklı fonksiyonel gruba sahip SAN monomeri (0.24 g, $0.001 \mathrm{~mol}) 0.1 \mathrm{M} \mathrm{KOH}$ çözeltisinde çözüldü ve 50 ml'lik üç boyunlu bir balona alındı. Polimerizasyon reaksiyonu termometre, manyetik karıştırıcı ve içinde $\mathrm{NaOCl}$ çözeltisi bulunduran bir damlatma hunisi eşliğinde geri soğutuculu sistemde gerçekleştirildi. Reaksiyon tamamlandıktan sonra karışım oda sıcaklığına kadar soğutuldu ve karışımdaki baza eşdeğer miktarda $\mathrm{HCl}$ ile nötrleştirildi. Çöken ürünler süzülerek ayrıldı ve 3'er kez 50 ml'lik sıcak suyla yıkanarak mineral tuzlardan arındırıldı. Oluşan polisulfoaminonaftol polimeri [PSAN] etüvde 110 ${ }^{\circ} \mathrm{C}$ de kurutuldu [9].

FT-IR (v, cm-1). 3408, $3262(\mathrm{OH} / \mathrm{NH}$, intramoleküler veya intermoleküler hidrojen bağı), $1643(\mathrm{C}=\mathrm{N})$, 1614, 1587 (Ar), 1307 (C-N), 1381 (-OH), 1218 (C- 
O), 1162, $1033\left(-\mathrm{SO}_{3}\right), 815,738 \mathrm{~cm}^{-1}$ (-CH substitüe naftalen halkası için). ${ }^{1} \mathrm{H}-\mathrm{NMR}$ (DMSO-d $)$ ): $\delta \mathrm{ppm}$, $10.63(\mathrm{~s}, \mathrm{Hh}, 1 \mathrm{H}), .32(\mathrm{~s}, \mathrm{Hb}, 1 \mathrm{H}), 7.20(\mathrm{~s}, \mathrm{Hc}, 1 \mathrm{H})$, $7.07(\mathrm{~s}, \mathrm{Hd}, 1 \mathrm{H}) .{ }^{13} \mathrm{C}-\mathrm{NMR}$ (DMSO-d 6$): \delta \mathrm{ppm}$, 149.87(C4), 144.62 (C7), 139.67 (C6), 130.47 (C2), 127.14 (C8), 126.12(C10), 124.69 (C9), 114.69 (C3), 114.86 (C1), 110.49 (C5).

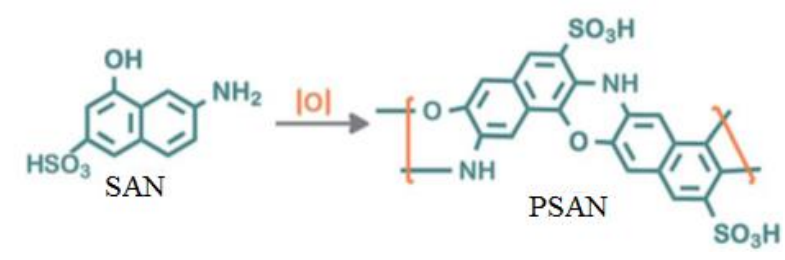

Şekil 1. PSAN için sentetik prosedür

\subsubsection{Blendlerin Hazırlanması}

İlgili blendler literatürün öngördüğü şekilde eriyik halde twin-screw microextruder'de hazırland1. Bunun için ilgili blendler, eriyik halde saf poliolefin malzemeye (polipropilen) $60 \mathrm{rpm}$ de 210 ${ }^{\circ} \mathrm{C}$ lik sıcaklıkta yaklaşık 45 dakikalık sürelerde PSAN in $\% 0.5, \% 1$ ve $\% 3$ oranlarında yüklenmesi ile elde edildi. Tüm blendler de extruderin çalışma koşulları çerçevesinde $4 \mathrm{gr}^{\prime}$ lık poliolefin temelli malzeme kullanıldı. Hazırlanan blendler $\mathrm{P} \gamma_{\mathrm{n}}$ olarak kodlandı (polipropilenin miktarına göre ağırlıkça \%0.5'lik PSAN ilavesinde P $\gamma_{1} ; \% 1$ PSAN ilavesinde $\mathrm{P} \gamma_{2} ; \% 3$ PSAN ilavesinde, $\mathrm{P} \gamma_{3}$ ) [10]

\subsection{Metot}

Çeşitli endüstriyel malzemelerin isısal stabilite ve bozunmalarına ilişkin kinetik parametrelerin tayininde genel olarak $i$-termogravimetrik analiz (TG), ii-diferansiyel termal analiz (DTA), iiidiferansiyel taramalı kalorimetre (DSC) ve ivderivative termogravimetri (DTG) teknikleri kullanılmaktadır. Bu yöntemler içerisinde, ısısal bozunma kinetiği parametrelerinin tayininde en yaygın kullanılan TG tekniğidir. Bu teknikte isıtma işlemi, ya sabit sıcaklıkta (izotermal) ya da sıcaklığın zamanla lineer olarak değişimiyle (nonizotermal) gerçekleştirilmektedir. Her iki isıtma prosedüründen yararlanılarak literatüre birçok kinetik modeller kazandırılmış ve bu modeller Flynn ve Wall [11] tarafından iintegral yöntemler, ii-diferansiyel yöntemler, iiifark diferansiyel yöntemler, iv-başlangıç hızına uygulanan yöntemler ve $v$-non-lineer isitma hizı yöntemleri olarak sınıflandırılmıştır. Bunlardan başlangıç hızına uygulanan yöntemler ve nonlineer 1sıtma hızı yöntemleri literatürde genel olarak kabul görmemektedir. Bununla birlikte reaksiyon mertebesi, $n$, aktivasyon enerjisi, $E$ ve ön üstel faktör, $A$ gibi kinetik parametreler ve aktivasyon entropisi, $\Delta S^{*}$, aktivasyon entalpisi, $\Delta H^{\sharp}$ ve aktivasyon serbest enerjisi, $\Delta G^{\sharp}$ gibi termodinamik parametreler integral, diferansiyel ve fark diferansiyel yöntemlerde kullanılan kinetik modellerin matematiksel ifadelerinden faydalanılarak tayin edilmektedir. Yukarıda isimleriyle anılan yöntemlerin dışında, literatürde pek çok kinetik modelleme önerilmekte ve herhangi bir materyalin kinetik ve termodinamik parametrelerinin tayininde bu kinetik modellerden uygun olan bir veya bir kaçı kullanılmaktadır. İncelenmek istenen herhangi bir organik veya inorganik madde için her kinetik yöntem uygulanamamakta ve kinetik yöntem seçimi zorunda kalınmaktadır. Bu çalışmada sentetik bir prosedür izlenerek optimum şartlar altında elde edilen PSAN /poliolefin temelli blendlerin katı hal 1sisal bozunma kinetiklerinin "thermal analysis 1.00" isimli PC programı [12] kullanılarak en uygun kinetik yönteme göre hesaplanması amaçlanmıştır.

\subsubsection{Kinetik Denklemler}

Katı hal reaksiyonlarının mekanizmaları ve kinetik çalışmaları için çoğunlukla non-isotermal metotlar kullanılır. $\mathrm{Bu}$ termoanalitik metotların çoğu Arrhenius denklemine, $\mathrm{k}=A \exp (-E / \mathrm{R} T)$, dayanır ve hiz ifadesi, $\mathrm{d} \alpha / \mathrm{d} t=\mathrm{kf}(\alpha)$ olarak verilir. Burada $\mathrm{f}(\alpha)$ tepkime mekanizmasına bağlı kinetik fonksiyon, $\alpha$ ise tepkime süresince katı reaktantlardaki 0-1 aralığında değişen dönüşüm kesridir. $\mathrm{f}(\alpha)=(1-\alpha)^{\mathrm{n}}$ ve sabit sıcaklıkta $\mathrm{d} T / \mathrm{d} t=\beta$ eşit olursa hız ifadesi aşağıdaki şekilde yazılabilir.

$$
\mathrm{g}(\alpha)=\int_{0}^{\alpha}\left[\frac{1}{(1-\mathrm{n})^{\mathrm{n}}}\right] \mathrm{d} \alpha=\frac{\mathrm{A}}{\beta} \int_{\mathrm{T}_{0}}^{\mathrm{T}} \exp \left(\frac{-\mathrm{E}}{\mathrm{RT}}\right) \mathrm{dT}
$$

Burada; $n$ tepkime mertebesidir. Buradan $n=1$ için denklem (1) ve $n \neq 1$ değerler için denklem (2) yazılabilir.

$$
\begin{gathered}
\int_{0}^{\alpha}\left[\frac{1}{(1-\mathrm{n})^{\mathrm{n}}}\right] \mathrm{d} \alpha=-\ln (1-\alpha) \\
\int_{0}^{\alpha}\left[\frac{1}{(1-\mathrm{n})^{\mathrm{n}}}\right] \mathrm{d} \alpha=-\frac{1-(1-\alpha)^{1-\mathrm{n}}}{1-\mathrm{n}}
\end{gathered}
$$


Denklem (3)'ün çözümü için farklı yaklaştırmaların kullanıldığı pek çok teknik vardır. Aşağıda verilen ve PSAN/polipropilen blendlerinin katı hal bozunma kinetiklerinin hesaplamalarında kullanılan yöntemler bunlardan bir kaçıdır.

\subsubsection{Coats-Redfern Yöntemi [13]}

Coats ve Redfern denklem (3)'ün çözümü için Doyle [14] yaklaştırmasını kullanmışlar ve denklem (4)' ü elde etmişlerdir.

$$
\ln \left(\frac{\mathrm{g}(\alpha)}{\mathrm{T}^{2}}\right)=\ln \left(\frac{\mathrm{AR}}{\mathrm{E} \beta}\left(1-\frac{2 \mathrm{RT}}{\mathrm{E}}\right)\right)-\frac{\mathrm{E}}{\mathrm{RT}}
$$

Denklem (4)' e göre $\ln \left(g(\alpha) / \mathrm{T}^{2}\right)$ ye karşı $1000 / \mathrm{T}$ arasında çizilen grafik eğimi - E/R olan düz bir çizgi verir.

\subsubsection{MacCallum-Tanner yöntemi [15]}

$$
\log (g(\alpha))=\log \frac{\mathrm{AE}}{\beta \mathrm{R}}-0.4828 \mathrm{E}^{0.4351}-\frac{449+217 \mathrm{E}}{\mathrm{T} .10^{3}}
$$

Denklem (5)'e göre $\operatorname{logg}(\alpha)^{\prime}$ ye karşı 1000/T grafiğinin eğiminden aktivasyon enerjisi, ordinatı kesim noktasından ön-üstel faktör bulunabilir

\subsubsection{Horowitz-Metzger Yöntemi [16]}

$\mathrm{Bu}$ yöntem, karakteristik bir sicaklık ve parametreyi aşağıdaki denklemle tanımlar.

$$
\ominus=\mathrm{T}-\mathrm{Tm}
$$

Eğer reaksiyon mertebesi 1 olarak alınırsa karakteristik bir sicaklik $(1-\alpha)_{\mathrm{m}}=1 / \mathrm{e}=0.368$ de belirlenebilir ve aşağıdaki gibi bir denklem elde edilir.

$$
\ln \ln (\mathrm{g}(\alpha))=\frac{\mathrm{E} \theta}{\mathrm{RR}_{\mathrm{m}}^{2}}
$$

Şayet reaksiyon mertebesi bilinmiyorsa karakteristik bir sicaklık maksimum 1sitma hiz1 için belirlenebilir. $\Theta=0,(1-\alpha)=(1-\alpha) \mathrm{m}$ ve $(1-\alpha) \mathrm{m}=\mathrm{n}^{1 / 1}$ n olursa aşağıdaki denklem elde edilir.

$$
\ln (\mathrm{g}(\alpha))=\ln \frac{\mathrm{ART}_{\mathrm{m}}^{2}}{\beta \mathrm{E}^{2}}-\frac{\mathrm{E}}{\mathrm{RT}_{\mathrm{m}}}+\frac{\mathrm{E} \theta}{\mathrm{RT}_{\mathrm{m}}^{2}}
$$

Denklem (7)'e göre $\operatorname{lng}(\alpha)^{\prime}$ ya karşı $\Theta^{\prime}$ nın grafiğinden aktivasyon enerjisi hesaplanabilir

\subsubsection{4. van Krevelen Yöntemi [17]}

Termogravimetrik verilerden ilk ciddi teoriksel ifadeyi van Krevelen ve ark yapmışlardır. Yazar aşağıdaki son denklemi elde etmek için exponential integral için empirik bir yaklaştırma kullanmıştır.

$$
\begin{aligned}
& \log (g(\alpha))=\mathrm{B}+\left(\frac{\mathrm{E}}{\mathrm{RT}_{\mathrm{r}}}+1\right) \log \mathrm{T} \\
& \mathrm{B}=\frac{\mathrm{A}}{\beta}\left(\frac{\mathrm{E}}{\mathrm{RT}_{\mathrm{r}}}+1\right)^{-1}\left(\frac{0.386}{\mathrm{~T}_{\mathrm{r}}}\right)^{\mathrm{E} / \mathrm{RT}_{\mathrm{r}}}
\end{aligned}
$$

Burada Tr referans sicaklığıdır. Denklem (9)’a göre $\operatorname{logg}(\alpha)^{\prime}$ nın $\log \mathrm{T}$ ye karşı çizilen grafiğin eğiminden aktivasyon enerjisi bulunabilir.

\subsubsection{Wanjun-Yuwen-Hen-Cunxin Yöntemi [18]}

$$
\ln \left(\frac{\mathrm{g}(\alpha)}{\mathrm{T}^{1.8946}}\right)=\left[\ln \frac{\mathrm{AR}}{\beta \mathrm{E}}+3.6350-1.89461 \ln \mathrm{E}\right]-1.0014\left(\frac{\mathrm{E}}{\mathrm{RT}}\right)
$$

Denklem (10)'a göre $\ln \left(g(\alpha) / \mathrm{T}^{1.8946}\right)$ ye karşı $1 / \mathrm{T}$ arasında çizilen grafikten eğimi -E/R olan düz bir çizgi elde edilir. Ayrıca ordinatı kesim noktasından ön-üstel faktör bulunabilir.

\subsubsection{Madhusudanan-Krishnan-Ninan Yöntemi} [19]

$$
\ln \left(\frac{\mathrm{g}(\alpha)}{\mathrm{T}^{1.9206}}\right)=\left[\ln \frac{\mathrm{AR}}{\beta \mathrm{E}}+3.7678-1.92061 \quad \ln \mathrm{E}\right]-0.12040\left(\frac{\mathrm{E}}{\mathrm{RT}}\right)
$$

Denklem (11)'e göre $\mathrm{g}(\alpha) / \mathrm{T}^{1.9206}$ ye karşı $1 / \mathrm{T}$ arasında çizilen grafiğin eğimi aktivasyon enerjisini, ordinatı kestiği noktada ön-üstel faktörü verir.

\section{BULGULAR ve TARTIŞMA}

\subsection{Isisal analizler}

$\mathrm{P} \gamma_{\mathrm{n}}$ blendlerinin 1sisal analizleri TG/DTG-DTA ölçümleri vasıtasıyla belirlendi. TG/DTG-DTA analizleri, 8-10 mg lik numunelerle platin kaplarda dinamik azot gazı altında $60 \mathrm{~mL} / \mathrm{dk}$ akış hızında $1000{ }^{\circ} \mathrm{C}$ ye kadar 1sitilarak izlendi. Kinetik hesaplamalar için 1sıtma hıları 5, 10, 15 ve 20 ${ }^{\circ} \mathrm{C} / \mathrm{dk}$ olarak seçildi. Detaylı bir kinetik analiz için tüm analizler tekrarlanabilir sonuçlar elde edilinceye kadar yenilendi. Analizlerde referans materyal olarak $\mathrm{Al}_{2} \mathrm{O}_{3}$ kullanıldı.

\subsection{PSAN ile katkılandırılmış polipropilen blendlerinin TG/DTG-DTA analizleri}

Şekil 2 saf PP ve P $\gamma_{\mathrm{n}}$ blendlerinin $5^{\circ} \mathrm{C} / \mathrm{dk}^{\prime}$ lik 1 sıtma hızlarındaki TG/DTG-DTA analizlerini göstermektedir. Buna göre TG/DTA-DTA eğrileri $\mathrm{PP} \quad$ ve $\mathrm{P} \gamma_{\mathrm{n}}$ blendlerinin tek basamakta bozunduğunu gösterdi. Ayrıca PP, $\mathrm{P} \gamma_{0,5}, \mathrm{P} \gamma_{1}$ ve $\mathrm{P} \gamma_{3}$ 
blendlerinin, bozunmaya başlama sıcaklıkları sirasiyla $431{ }^{\circ} \mathrm{C}, 434{ }^{\circ} \mathrm{C}, 435{ }^{\circ} \mathrm{C}$ ve $436{ }^{\circ} \mathrm{C}$ olarak okundu. İlave PSAN katkı polimerinin miktarı artıkça PP in bozunmaya başlama sicaklığının yüksek sıcaklıklara kaydığı ve termal karalılığının artığı görüldü.

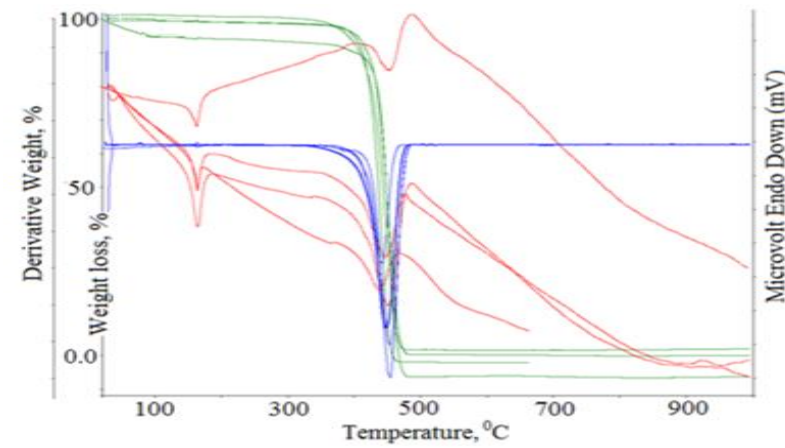

Şekil 2. Saf polipropilen ve $\mathrm{P} \gamma_{\mathrm{n}}$ blendlerinin $5{ }^{\circ} \mathrm{C} / \mathrm{dk}^{\prime} \mathrm{l}_{\mathrm{l}}$ ısıtma hızlarındaki termogramları

DTA eğrilerinde yaklaşı $163{ }^{\circ} \mathrm{C}$ ve $436{ }^{\circ} \mathrm{C}$ lerde görülen endotermik etkiler sirasıyla PP in erime noktasina ve blendlerin katı hal bozunma reaksiyonlarına atfedildi. DTG eğrilerinden en yüksek bozunma hızına karşılık gelen sıcaklık değerleri $\mathrm{PP}, \quad \mathrm{P} \gamma_{0.5,} \mathrm{P} \gamma_{1}$ ve $\mathrm{P} \gamma_{3}$ blendleri için sirasiyla $442^{\circ} \mathrm{C}, 447{ }^{\circ} \mathrm{C}, 448{ }^{\circ} \mathrm{C}$ ve $450{ }^{\circ} \mathrm{C}$ olarak okundu. Bununla birlikte $\mathrm{P} \gamma_{\mathrm{n}}$ blendlerinin DTA eğrilerinden $\mathrm{PP}^{\prime}$ ne ait vicat yumuşama noktası okunamadi. Diğer yandan Şekil 3 'de PSAN polimeri ile katkılandırılmış PP blendlerinin farklı isıtma hizlarındaki $\left(5,10,15\right.$ ve $\left.20^{\circ} \mathrm{C} / \mathrm{dk}\right) \mathrm{TG} / \mathrm{DTG}-$ DTA termogramları verildi. Buna göre $P \gamma_{n}$ blendlerinin tümünde yalnızca tek bir bozunma basamağı görüldü. Ayrıca ısıtma hızının artışı ile başlangıç bozunma sıcaklıklarının daha yüksek sıcaklıklara kaydığı belirlendi. Elde edilen tüm termogramların benzer karakterde oldukları tespit edildi. P $\gamma_{\mathrm{n}}$ blendlerinin 5, 10, 15 ve $20{ }^{\circ} \mathrm{C} / \mathrm{dk} l \mathrm{lk}$ ısıtma hızlarında gerçekleştirilen katı hal isısal bozunma basamağ 1 için en yüksek bozunma hızına karşılık gelen sıcaklık değerleri DTG eğrilerinden sırası ile $\mathrm{P} \gamma_{0.5}$ blendi için 434, 447, 453 ve $461{ }^{\circ} \mathrm{C}$, $\mathrm{P} \gamma_{1}$ blendi için $435,447,457$ ve $461{ }^{\circ} \mathrm{C}$ ve $\mathrm{P} \gamma_{3}$ blendi için $436,450,458$ ve $462{ }^{\circ} \mathrm{C}$ olarak okundu. Buna göre tüm termogramların beklenildiği gibi artan 1sıtma hızıyla yüksek sıcaklık değerlerine kaydığı tespit edildi.
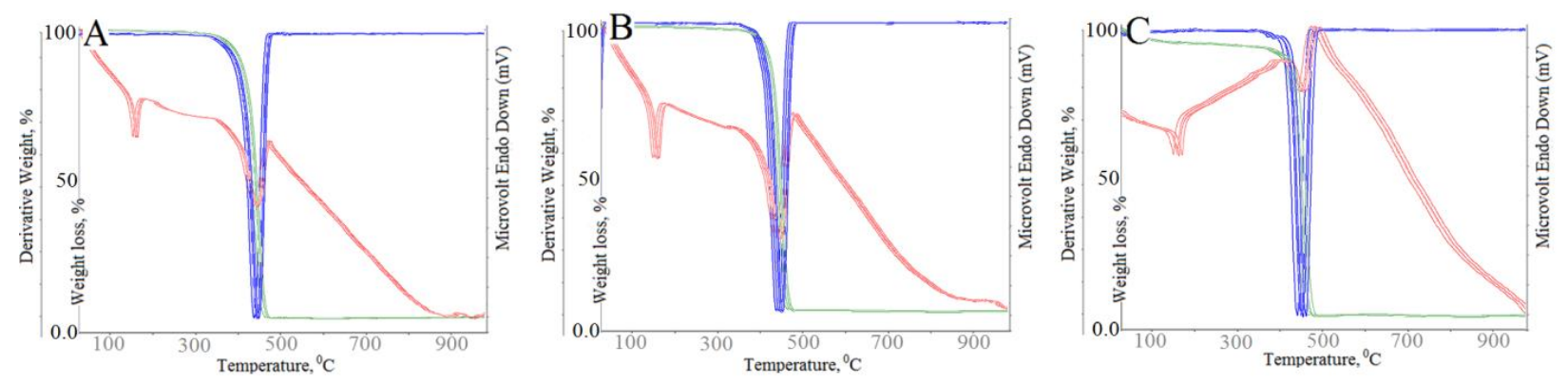

Şekil 3. $P \gamma_{0.5}$ (a), $P \gamma_{1}$ (b) ve $P \gamma_{3}$ (c) blendlerinin farklı 1sıtma hızlarındaki tipik dinamik TG/DTG ve DTA termogramları

\subsection{PSAN ile katkılandırılmış PP blendlerinin katı hal bozunma kinetikleri}

PSAN polimeri ile katkılandırılmış PP blendlerinin isısal bozunmasina ait kinetik parametrelerinin hesaplanması, tek bir isıtma hizına dayanan integral (CR, WYHC ve MKN), diferansiyel (HM ve vK) ve fark-diferansiyel yöntemler (MT) kullanılarak yapıldı. Ayrıca aktivasyon enerjisinin ısıtma hızına bağımlılığı ve blendlerin katı hal 1sisal bozunmasinı tanımlayabilecek en uygun yöntem araştırıldı. Bu amaçla blendlerin isısal bozunma basamağını tanımlayabilecek aktivasyon enerjisi, reaksiyon mertebesi ve ön-üstel faktör gibi bazı kinetik parametrelerin yanında aktivasyon entalpisi, aktivasyon serbest enerjisi ve aktivasyon entropisi gibi bazı termodinamik parametreler tek bir ısıtma hızına dayanan yöntemler kullanılarak hesapland1.

Tablo 1. $\mathrm{P} \gamma_{\mathrm{n}}$ blendleri için tek bir ısıtma hızına dayanan yöntemler vasıtasıyla elde edilen kinetik parametreler 


\begin{tabular}{|c|c|c|c|c|c|c|c|c|c|}
\hline Blend & $\beta$ & Metot & $\mathrm{n}$ & $E$ & $\ln A$ & $\Delta S^{\neq}$ & $\Delta \mathrm{H}^{\mp}$ & $\Delta \mathrm{G}^{\neq}$ & $\mathrm{r}$ \\
\hline \multirow{4}{*}{$\mathrm{P} \gamma_{\mathrm{n} 0.5}$} & $5{ }^{\circ} \mathrm{C} / \mathrm{dk}$ & WHYC & 2.0 & 209.09 & -36.27 & -583.37 & 308.6 & 462.8 & 0.96579 \\
\hline & 10 & $\mathrm{CR}$ & 2.0 & 209.98 & -35.88 & -585.74 & 304.3 & 458.7 & 0.98718 \\
\hline & 15 & $\mathrm{CR}$ & 2.1 & 210.78 & -33.93 & -588.06 & 296.8 & 450.7 & 0.97809 \\
\hline & 20 & WHYC & 2.2 & 212.31 & -46.47 & -591.57 & 281.0 & 442.2 & 0.99768 \\
\hline \multirow{4}{*}{$P \gamma_{n 1}$} & $5{ }^{\circ} \mathrm{C} / \mathrm{dk}$ & $\mathrm{CR}$ & 1.9 & 209.75 & -39.43 & -624.53 & 234.8 & 503.6 & 0.95367 \\
\hline & 10 & MKN & 2.0 & 211.57 & -38.85 & -626.08 & 230.1 & 493.0 & 0.97688 \\
\hline & 15 & WHYC & 2.1 & 213.68 & -36.99 & -628.84 & 224.3 & 488.7 & 0.97678 \\
\hline & 20 & MKN & 2.1 & 215.09 & -35.74 & -631.98 & 218.0 & 481.4 & 0.97868 \\
\hline \multirow{4}{*}{$P \gamma_{n} 3$} & $5^{\circ} \mathrm{C} / \mathrm{dk}$ & WHYC & 1.7 & 212.39 & -44.08 & -862.88 & 103.7 & 93.67 & 0.99777 \\
\hline & 10 & $\mathrm{CR}$ & 1.8 & 214.74 & -43.33 & -869.91 & 101.3 & 88.00 & 0.97733 \\
\hline & 15 & WHYC & 1.9 & 215.22 & -41.70 & -876.78 & 99.96 & 82.35 & 0.98766 \\
\hline & 20 & WHYC & 1.9 & 217.08 & -40.74 & -883.31 & 93.74 & 73.73 & 0.98778 \\
\hline
\end{tabular}

n-reaksiyon derecesi, $\beta$ - 1sıtma hızı, E-aktivasyon enerjisi, A-ön-üstel faktör, $\Delta S^{\neq}$-aktivasyon entropi değişimi, $\Delta H^{z_{-}}$ aktivasyon entalpi değişimi, $\Delta G^{\ddagger}$-aktivasyon serbest enerji değişimi, $r$-regresyon katsayısı

Thermal analysis 1.00 isimli PC programı kullanılarak elde edilen kineitk ve termodinamik veriler Tablo 1'de özetlendi. Tablo 1 den $P \gamma_{n}$ blendlerinin katı hal isısal bozunma kinetiklerinin hesaplanmasında regresyon katsayılarına göre en uygun yöntemlerin, genellikle integral yöntemler (WHYC, MKN ve CR yöntemleri) olduğu görüldü. Tüm blendlerin isısal bozunma basamağı için elde edilen regresyon katsayıları $0.1<\alpha<0.8$ aralığında oldukça yüksek (1 yakın) elde edildi. Ayrıca MT ve HM yöntemlerinden elde edilen Arrhenius eğrilerinin kolerasyon çarpıklıkları oldukça fazla olduğundan tabloya dâhil edilmedi. Bununla birlikte Tablo 1 den 5 ve $20^{\circ} \mathrm{C} / \mathrm{dk}$ lik 1sitma hızlarındaki katı hal bozunmalarıyla ilgili aktivasyon enerji değerlerinin $\mathrm{P} \gamma 0.5$ blendi için sırası ile 209.09 ve $212.31 \mathrm{~kJ} / \mathrm{mol}, \quad \mathrm{P} \gamma 1$ blendi için 209.75 ve $215.09 \mathrm{~kJ} / \mathrm{mol}$ ve $\mathrm{P} \gamma_{3}$ blendi için ise 212.39 ve $217.08 \mathrm{~kJ} / \mathrm{mol}$ olduğu görüldü. Buradan tüm blendlerin katı hal isisal bozunmalariyla ilgili aktivasyon enerji değerlerinin artan 1sıtma hızıyla orantılı olduğu rahatlıkla söylenebilir [20-21]. Ayrıca PP içerisindeki PSAN polimerinin miktarındaki artışla tüm ısıtma hızlarında aktivasyon enerjisinin artığı görüldü. Bu durum PSAN polimerinin oksidant etkisinden dolayı PP zincirlerini kırarak çapraz bağlar oluşturmasına atfedildi. Diğer yandan $P \gamma_{n}$ blendlerinin katı hal isısal bozunma basağına ait reaksiyonun mertebesinin artan 1sıtma hızı orantılı olduğu bulundu (P $\gamma_{0.5}$ blendi için 5 o C/dk lik 1sıtma hızındaki reaksiyon mertebesi 2.0 iken $20{ }^{\circ} \mathrm{C} / \mathrm{dk}$ lik 1sıtma hızındaki reaksiyon mertebesi 2.2 dir). Yine Tablo 1 den $P \gamma_{n}$ blendlerinin katı hal 1sisal bozunmaya ait reaksiyon mertebelerinin (herbir 1sıtma hızı için ayrı ayrı değerlendirildiğinde) PP içerisinde artan PSAN miktarıyla ters orantılı bir şekilde değiştiği bulundu. Buna göre $\mathrm{P} \gamma_{0.5}, \mathrm{P} \gamma_{1}, \mathrm{P} \gamma_{3}$ blendleri için $5{ }^{\circ} \mathrm{C} / \mathrm{dk}$ lik isıtma hızındaki reaksiyon mertebeleri sirasi ile 2.0, 1.9 ve 1.7 dir. Literatüre göre herhangi bir materyalin katı hal 1sısal bozunma basamağına ait kinetik tripletlerden ( $E, A$ ve $n$ ) reaksiyon mertebesinin 1sitma hizı veya gaz akış hızı gibi bazı parametrelerden etkilenmesi fiziksel olarak bir anlam ifade etmemektedir [2223]. Kinetik ve termodinamik hesaplamalarda $n$, $A, S, H, G, E$ ve $r$ ilgili blendlerin isisal bozunma basamağına karşılık gelen Arrhenius eğrilerinin eğimlerinden hesaplandı.

$\mathrm{P} \gamma_{\mathrm{n}}$ blendlerinin 1sisal kat1 hal bozunma basamağına ait termodinamik tripletler (aktivasyon entropi değişimi, $\Delta S^{\neq}$, aktivasyon entalpi değişimi, $\Delta H^{\ddagger}$ ve aktivasyon serbest enerji değişimi, $\Delta G^{\neq}$) denklem (12) ve (13) vasitasıyla bulundu [24].

$$
\begin{gathered}
\Delta S^{\#}=2.303 \log (A h / k T) R \\
\Delta H^{\neq}=E-R T, \Delta G^{\#}=\Delta H^{\neq}-T
\end{gathered}
$$

Burada $h$ ve $T$ sirasiyla Planck sabiti ve mutlak sıcaklıktır. Çalışılan dönüşüm değerleri içerisinde Tablo 1 deki aktivasyon entropisinin negatif çıkması $\mathrm{P} \gamma_{\mathrm{n}}$ blendlerinin katı hal 1sısal bozunma basamağına ait reaksiyon hızının normalden daha hızlı olduğunu gösterir.

\section{SONUÇ}

PSAN polimeri ile katkılandırılmış PP blendlerinin 1sisal bozunmasına ait kinetik parametrelerinin 
hesaplanması, tek bir 1sitma hizina dayanan integral, diferansiyel ve fark-diferansiyel yöntemlerden CR, HM, VK, WYHC, MKN ve MT yöntemleri kullanılarak gerçekleştirildi. $5{ }^{\circ} \mathrm{C} / \mathrm{dk}^{\prime} \mathrm{l}$ k 1sıtma hızında bozunmaya başlama sıcaklıkları sırasıyla PP için $431^{\circ} \mathrm{C}$, P $\gamma 0.5$ blendi için $434^{\circ} \mathrm{C}, \mathrm{P} \gamma /$ blendi için $435^{\circ} \mathrm{C}$ ve $\mathrm{P} \gamma 3$ blendi için $436^{\circ} \mathrm{C}$ olarak bulundu. Buna göre artan PSAN miktarı ile PP blendlerinin ısısal kararlılığının artığı görüldü. Tüm blendler için farklı ısıtma hızlarında elde edilen termogramların benzer karakterde oldukları ve bundan dolayı da benzer bozunma reaksiyonları gösterdikleri belirlendi. PSAN/PP blendlerinin katı hal isısal bozunma kinetiklerinin hesaplanmasında kinetik yöntemler regresyon katsayılarına göre siralandı ve isısal bozunma kinetiği için en uygun yöntemler belirlendi. Buna göre 1sısal bozunmayı en iyi integral yöntemlerin (WHYC, MKN ve CR yöntemleri) betimlediği bulundu. Çalışılan $0.1<\alpha<0.8$ aralığında Arrhenius eğrilerinden blendlerin bozunması için gerekli olan aktivasyon enerji değerlerinin yaklaşık 210$220 \mathrm{~kJ} / \mathrm{mol}$ arasında olması gerektiği hesaplandı.

Teşekkür: $\mathrm{Bu}$ çalışma Türkiye Bilimsel ve Teknolojik Araştırma Kurumu, TUBITAK-KBAK tarafından $113 Z 587$ nolu proje ile desteklenmiştir.

\section{Referanslar}

[1] Şirin, K.;Yavuz, M.;Çanlı, M. The Influence of Dilauroyl Peroxide on Mechanical and Thermal Properties of Different Polypropylene Matrices. Polymer (Korea), 2015; 39(2), 1-10

[2] Şirin, K.; Doğan, F.;Çanlı, M.;Yavuz M. Mechanical Properties of Polypropylene (PP)+High-Density Polyethylene (HDPE) Binary Blends: Non-isothermal Degradation Kinetics of PP + HDPE (80/20) Blends. Polymers for Advanced Technologies, 2013; 24(8), 715-722.

[3] Doğan, F.; Şirin, K.; Kaya, İ.Balcan M. The Influence of $\mathrm{CaCO}_{3}$ Filler Component on Thermal Decomposition Processof PP/LDPE/ DAP Ternary Blend. Polymers for Advanced Technologies, 2010; 21(7), 512-519

[4] Şirin, K.;Çengel, Ö.;Canli, M. Thermal and Mechanical Propertiesof LDPE by The Effects of Organic Peroxides: Mechanical Properties of
LDPE. Polymers for Advanced Technologies, 2017, DOI: 10.1002/pat.3992

[5] Zsoldos, G.;Kollár, M. Structural Analysis of Polyolefin-Poly(MethylMethacrylate) Blends. Journal of Thermal Analysis and Calorimetry, 2014; 119(1), 63-72

[6] Contat-Rodrigo, L.;Amparo, R. G.;Imrie, C. Thermal Analysis of High-Density Polyethylene and Low-Density Polyethylene with Enhanced Biodegradability. Journal of Applied Polymer Science, 2002; 86(3), 764-772

[7] Schartel, B.;Braun, U.;Schwarz, U.;Reinemann, S. Fire Retardancy of Polypropylene/flax Blends. Polymer, 2003; 44, 6241-6250.

[8] Cai, Q.;Wei, F.;Huang, S.; Lin, F.;Chen, W. G.Thermal Stability, Latent Heat and Flame Retardant Properties of TheThermal Energy Storage Phase Change Materials Based on Paraffin/High Density Polyethylene Composites. Renewable Energy, 2009; 34:2117-2123.

[9] Doğan, F.; Temizkan,K.; Kaya, İ. Regioselective Synthesis of Polygamma (g) acid, RSC Advances, 2015; 5, 53369.

[10] Doğan, F. Elektro-Aktif Flüoresant Polimerlerin Poliolefin Temelli Malzemelerde Antistatik Materyal Olarak Kullanımı, TUBITTAKKBAK Projesi, 2016; Proje No.113Z587.

[11] Flynn, J.; Wall, L. A Quick, Direct Method for The Determination of Activation Energy from Thermogravimetric Data. Journal of Polymer Science Part B: Polymer Letter, 1966; 4, 323-328.

[12] Doğan, F. Termogravimetrik Verilerin Kinetik Analiz Yöntemlerine İlişkin Bilgisayar Programı Geliştirilmesi ve Polimerlerin Isısal Degradasyon ve Bozunma Kinetiğine Uygulanması, Doktora Tezi, 2006; Ege Üniversitesi, Izmir, Turkiye,

[13] Coats, A. W.;Redfern, J. P. Kinetics Parameters fromThermogravimetric Data. Nature, 1964; 201, 68-69 
[14] Doyle, D. C. Synthesis and Evaluation of Thermally Stable Polymers. II. Polymer Evaluation. Journal of Applied Polymer Science,1961; 5, 285

[15] Maccallum, J.R.;Tanner, J. Derivation of Rate EquationsUsed in Thermogravimetry. Nature, 1970 ; 225(5238), 1127-1128

[16] Horowitz, H. H.;Metzger, G. Interpretation of Thermogravimetric Traces. Fuel, 1963; 42(5), 418420.

[17] van Krevelen, D. W.;van Heerden, C.;Huntjons, F. Physicochemical Aspects of the Pyrolysis of Coal and Related Organic Compounds. Fuel,1951; 30, 253-259

[18] Wanjun, T.; Yuwen, L.;Hen, Z.; Cunxin, W. New Approximate Formula for Arrhenius Temperature İntegral. Thermochimica Acta, 2003; 408(1-2), 39-43

[19] Madhusudanan, P. M.;Krishnan, K. K.;Ninan, N. New Equations for Kinetic Analysis of Nonİsothermal Reactions. Thermochimica Acta, 1993; 221(1), 13-21.
[20] Doğan, F.; Ulusoy, M.; Ozturk, O. F.; Kaya, I.; Salih,B. N,N'-Bis(3,5-di-t-butylsalicylideneimine) propanediamine and its SomeTetradentate Schiff Base Complexes. Journal of Thermal Analysis and Calorimetry, 2009; 96, 267.

[21] Doğan, F.; Dayan, O.; Yürekli, M.; Çetinkaya, B. Thermal Study of Ruthenium(II) Complexes Containing Pyridine-2,6-Diimines. Journal of Thermal Analysis and Calorimetry, 2008; 91, 943.

[22] Doğan, F.;Gülcemal, S.; Yürekli, M.; Çetinkaya, B. Thermal Analysis Study of Imidazolinium and Some Benzimidazolium Salts by Tg. Journal of Thermal Analysis and Calorimetry, 2008; 91, 395.

[23] Vyazovkin, S.;Modification of The İntegral İsoconversional Method to Account for Variationin The Activation Energy. Journal of Computional Chemistry, 2001; 22, 178-183.

[24] Allan, J.R.;Bonner, J.G.;Gerrard, D.L.;Birnie, J. Structural Characterisation and Thermal Analysis Studies of The Compounds of Manganese(II), Cobalt(II), Nickel(II), Copper(II) and Zinc(II) with Poly(CrylicAcid), Thermochimica Acta. 1991; 185(2), 295-302. 